\title{
THE NUMBER OF UNITARILY $k$-FREE DIVISORS OF AN INTEGER
}

\author{
D. SURYANARAYANA AND R. SITA RAMA CHANDRA RAO
}

(Received 20 December 1972)

Communicated by G. Szekeres

\section{Introduction}

Let $k$ be a fixed integer $\geqq 2$. A positive integer $n$ is called unitarily $k$-free, if the multiplicity of each prime factor of $n$ is not a multiple of $k$; or equivalently, if $\boldsymbol{n}$ is not divisible unitarily by the $k$-th power of any integer $>1$. By a unitary divisor, we mean as usual, a divisor $d>0$ of $n$ such that $(d, n / d)=1$. The integer 1 is also considered to be unitarily $k$-free. The concept of a unitarily $k$-free integer was first introduced by Cohen (1961); $\$ 1)$. Let $Q_{k}^{*}$ denote the set of unitarily $k$-free integers. When $k=2$, the set $Q_{2}^{*}$ coincides with the set $Q^{*}$ of exponentially odd integers (that is, integers in whose canonical representation each exponent is odd) discussed by Cohen himself in an earlier paper $(1960 ; \S 1$ and \$6). A divisor $d>0$ of the positive integer $n$ is called a unitarily $k$-free divisor of $n$ if $d \in Q_{k}^{*}$. Let $\tau_{(k)}^{*}(n)$ denote the number of unitarily $k$-free divisors of $n$.

In this paper we prove the following.

THEOREM 1. For $x \geqq 3$,

(1.1) $\sum_{n \leq x} \tau_{(k)}^{*}(n)=\alpha_{k} x\left(\log x+2 \gamma-1+\frac{\zeta^{\prime}(k)}{\zeta(k)}+\sum_{p} \frac{(2 k p-k-1) \log p}{p^{k+1}-2 p+1}\right)+\Delta_{(k)}^{*}(x)$, where $\Delta_{(k)}^{*}(x)=O\left(x^{1 / k} \exp \left\{-A \log ^{3 / 5} x(\log \log x)^{-1 / 5}\right\}\right)$ or $O\left(x^{\alpha}\right)$, according as $k=2,3$ or $k \geqq 4 ; A$ being a positive constant, $\gamma$ is Euler's constant, $\alpha$ is the number which appears in the Dirichlet divisor problem (2.19) and $\alpha_{k}$ is the constant given by (2.1).

THEOREM 2. If the Riemann hypothesis is true, then for $x \geqq 3$, the error term $\Delta_{(k)}^{*}(x)$ in $(1.1)$ is given by $\Delta_{(k)}^{*}(x)=O\left(x^{(2-\alpha)(1+2 k(1-\alpha))} \omega(x)\right.$ or $O\left(x^{\alpha}\right)$, according as $k=2,3$ or $k \geqq 4$; where $\omega(x)=\exp \left\{A \log x(\log \log x)^{-1}\right\}, A$ being 
a positive constant and $\alpha$ is the number which appears in the Dirichlet divisor problem (2.19).

\section{Prerequisites}

Let $\mu(n)$ and $\phi(n)$ denote respectively the Möbius function and the Euler totient function. Let $\mu^{*}(n)$ denote the unitary analogue of the Möbius $\mu$-function defined by $\mu^{*}(n)=(-1)^{\nu(n)}$, where $\nu(n)$ is the number of distinct prime factors of $n$. Let $\sigma_{s}^{*}(n)$ denote the sum of the $s$-th powers of the square-free divisors of $n$. It is known Cohen (1961; Lemma 3.5), that

$$
\alpha_{k} \equiv \sum_{m=1}^{\infty} \frac{\mu^{*}(m) \phi(m)}{m^{k+1}}=\zeta(k) \prod_{p}\left(1-\frac{2}{p^{k}}+\frac{1}{p^{k+1}}\right),
$$

where the product is extended over all primes $p$ and $\zeta(k)$ is the Riemann Zeta function.

It can be easily shown by using standard arguments that

$$
\sum_{m \leqq x} \frac{\sigma_{-s}^{*}(m)}{m^{u}}=O\left(x^{1-u}\right) \text { for } s>0 \text { and } 0 \leqq u<1 .
$$

We need the following lemmas:

Lemma 2.1. (Suryanarayana and Sita Rama Chandra Rao (1975; Lemma 2.8)). For $x \geqq 3$ and for every $\varepsilon>0$,

$$
M_{n}^{*}(x) \equiv \sum_{\substack{m \leq x \\(m, n)=1}} \mu^{*}(m)=O\left(\sigma_{-1+\varepsilon}^{*}(n) x \delta(x)\right),
$$

where the $O$-constant is independent of $n$ and $x$ and $\delta(x)$ is given by

$$
\delta(x)=\exp \left\{-A \log ^{3 / 5} x(\log \log x)^{-1 / 5}\right\},
$$

$A$ being a positive constant.

Remark. Hereafter, all the constants implied by the $O$-symbols are independent of $n$ and $x$.

Lemma 2.2. (Suryanarayana and Sita Rama Chandra Rao (1975; Lemma 2.13)). For $x \geqq 3$,

$$
N^{*}(x) \equiv \sum_{m \leq x} \mu^{*}(m) \phi(m)=O\left(x^{2} \delta(x)\right),
$$

where $\delta(x)$ is given by (2.4).

Lemma 2.3 (Suryanarayana and Sita Rama Chandra Rao (to appear)) For $x \geqq 3$ and for every $\varepsilon>0$, 


$$
N_{n}^{*}(x) \equiv \sum_{\substack{m \leq x \\(m, n)=1}} \mu^{*}(m) \phi(m)=O\left(\sigma_{-1+\varepsilon}^{*}(n) x^{2} \delta(x)\right) .
$$

LEMMA 2.4. For $x \geqq 3, s>2$ and for every $\varepsilon>0$,

$$
\sum_{\substack{m>x \\(m, n)=1}} \frac{\mu^{*}(m) \phi(m)}{m^{s}}=O\left(\frac{\sigma_{-1+\varepsilon}^{*}(n) \delta(x)}{x^{s-2}}\right) .
$$

Proof. Putting $f(m)=1 / m^{s}$, it can be easily shown that

$$
f(m+1)-f(m)=O\left(\frac{1}{m^{s+1}}\right) .
$$

By partial summation and Lemma 2.3, we have

$$
\begin{aligned}
\sum_{\substack{m>x \\
(m, n)=1}} \frac{\mu^{*}(m) \phi(m)}{m^{s+1}} & =-N_{n}^{*}(x) f([x]+1)-\sum_{m>x} N_{n}^{*}(m)\{f(m+1)-f(m)\} \\
& =O\left(\frac{\sigma_{-1+\varepsilon}^{*}(n) \delta(x)}{x^{s-2}}\right)+O\left(\sum_{m>x} \frac{\sigma_{-1+\varepsilon}^{*}(n) \delta(m)}{m^{s-1}}\right) \\
& =O\left(\frac{\sigma_{-1+\varepsilon}^{*}(n) \delta(x)}{x^{s-2}}\right)+O\left(\sigma_{-1+\varepsilon}^{*}(n) \delta(x) \sum_{m>x} \frac{1}{m^{s-1}}\right),
\end{aligned}
$$

since $\delta(x)$ is monotonic decreasing. Also, since $s>2$,

$$
\sum_{m>x} \frac{1}{m^{s-1}}=O\left(\frac{1}{x^{s-2}}\right)
$$

Hence the lemma follows.

As a particular case of (2.7) for $n=1$, we have

$$
\sum_{m>x} \frac{\mu^{*}(m) \phi(m)}{m^{s}}=O\left(\frac{\delta(x)}{x^{s-2}}\right) .
$$

Lemma 2.5. For $x \geqq 3, s>2$ and for every $\varepsilon>0$,

$$
\sum_{\substack{m>x \\(m, n)=1}} \frac{\mu^{*}(m) \phi(m) \log m}{m^{s}}=O\left(\frac{\sigma_{-1+\varepsilon}^{*}(n) \delta(x) \log x}{x^{s-2}}\right) .
$$

Proof. Putting $g(m)=\log m / m^{s}$, it can be easily shown that

$$
g(m+1)-g(m)=O\left(\frac{\log m}{m^{s+1}}\right) .
$$

By partial summation, Lemma 2.3 and making use of the argument adopted in the proof of Lemma 2.4, we get (2.9).

As a particular case of (2.9) for $n=1$, we have 


$$
\sum_{m>x} \frac{\mu^{*}(m) \phi(m) \log m}{m^{s}}=O\left(\frac{\delta(x) \log x}{x^{s-2}}\right) .
$$

Lemma 2.6. For $s>2$,

$$
\sum_{\substack{m=1 \\(m, n)=1}}^{\infty} \frac{\mu^{*}(m) \phi(m)}{m^{s}}=\zeta(s-1) \prod_{p}\left(1-\frac{2}{p^{s-1}}+\frac{1}{p^{s}}\right) \prod_{p \mid n}\left\{\frac{p\left(p^{s-1}-1\right)}{p^{s}-2 p+1}\right\} .
$$

Proof. Let $e(m)=1$ or 0 according as $m=1$ or $m>1$. Then the series on the left becomes

$$
\sum_{m=1}^{\infty} \frac{\mu^{*}(m) \phi(m) e((m, n))}{m^{s}}
$$

This series is absolutely convergent for $s>2$ and the general term is a multiplicative function of $m$. Hence the series can be expanded into an infinite product of Euler type (Hardy and Wright (1960; Theorem 286)), so that we have

$$
\begin{aligned}
\sum_{\substack{m=1 \\
(m, n)=1}}^{\infty} \frac{\mu^{*}(m) \phi(m)}{m^{s}} & =\prod_{\substack{p \\
p \nmid n}}\left\{1-\sum_{i=1}^{\infty} \frac{p^{i-1}(p-1)}{p^{i s}}\right\} \\
& =\prod_{\substack{p \\
p \nmid n}}\left\{1-\frac{p-1}{p^{s}} \sum_{i=1}^{\infty} \frac{1}{p^{(i-1)(s-1)}}\right\}=\prod_{\substack{p \\
p \nmid n}}\left\{1-\frac{p-1}{p^{s}\left(1-\frac{1}{p^{s-1}}\right)}\right\} \\
& =\prod_{\substack{p \\
p \times n}}\left\{\frac{1-\frac{2}{p^{s-1}}+\frac{1}{p^{s}}}{1-p^{-(s-1)}}\right\} \\
& =\prod_{p}\left\{\frac{\left.1-\frac{2}{p^{s-1}}+\frac{1}{p^{s}}\right\} \cdot \prod_{p \mid n}\left\{\frac{\left.1-\frac{2}{p^{s-1}}+\frac{1}{p^{s}}\right\}^{-1}}{1-p^{-(s-1)}}\right\}^{-1}}{}\right. \\
& =\zeta(s-1) \prod_{p}\left(1-\frac{2}{p^{s-1}}+\frac{1}{p^{s}}\right) \prod_{p \mid n}\left\{\frac{p\left(p^{s-1}-1\right)}{p^{s}-2 p+1}\right\} .
\end{aligned}
$$

Hence the lemma follows.

As particular case of (2.11) for $n=1$, we have the following:

$$
\sum_{m=1}^{\infty} \frac{\mu^{*}(m) \phi(m)}{m^{s}}=\zeta(s-1) \prod_{p}\left(1-\frac{2}{p^{s-1}}+\frac{1}{p^{s}}\right) \text { for } s>2 .
$$

LEMMA 2.7. For $s>2$,

$$
\begin{aligned}
\sum_{m=1}^{\infty} \frac{\mu^{*}(m) \phi(m) \log m}{m^{s}}= & -\zeta(s-1) \prod_{p}\left(1-\frac{2}{p^{s-1}}+\frac{1}{p^{s}}\right) \\
& \times\left\{\frac{\zeta^{\prime}(s-1)}{\zeta(s-1)}+\sum_{p} \frac{(2 p-1) \log p}{p^{s}-2 p+1}\right\} .
\end{aligned}
$$


PRoof. This series is uniformly convergent for $s \geqq 2+\varepsilon>2$ and so by termwise differentiation of the series in (2.12) with respect to $s$, we get (2.13). For finding the derivative of the right hand side expression of (2.12) with respect to $s$, we write

$$
f(s)=\zeta(s-1) \prod_{p}\left(1-\frac{2}{p^{s-1}}+\frac{1}{p^{s}}\right) .
$$

Then

$$
\log f(s)=\log \zeta(s-1)+\sum_{p} \log \left(1-\frac{2}{p^{s-1}}+\frac{1}{p^{s}}\right)
$$

so that

$$
\frac{f^{\prime}(s)}{f(s)}=\frac{\zeta^{\prime}(s-1)}{\zeta(s-1)}+\sum_{p} \frac{(2 p-1) \log p}{\left(p^{s}-2 p+1\right)}
$$

and this gives

$$
f^{\prime}(s)=f(s)\left\{\frac{\zeta^{\prime}(s-1)}{\zeta(s-1)}+\sum_{p} \frac{(2 p-1) \log p}{\left(p^{s}-2 p+1\right)}\right\} .
$$

As a consequence of (2.8) and (2.12), we have

$$
\sum_{m \leq x} \frac{\mu^{*}(m) \phi(m)}{m^{k+1}}=\alpha_{k}+O\left(\frac{\delta(x)}{x^{k-1}}\right) .
$$

Similarly, as a consequence of (2.10) and (2.13), we have

$$
\begin{aligned}
\sum_{m \leq x} \frac{\mu^{*}(m) \phi(m) \log m}{m^{k+1}}=-\alpha_{k}\left\{\frac{\zeta^{\prime}(k)}{\zeta(k)}\right. & \left.+\sum_{p} \frac{(2 p-1) \log p}{\left(p^{k+1}-2 p+1\right)}\right\} \\
& +O\left(\frac{\delta(x) \log x}{x^{k-1}}\right),
\end{aligned}
$$

and as a consequence of (2.7) and (2.11) for $s=k+1$, we have by (2.1):

$$
\sum_{\substack{m \leq x \\(m, n)=1}} \frac{\mu^{*}(m) \phi(m)}{m^{k+1}}=\alpha_{k} \prod_{p \mid n}\left\{\frac{p\left(p^{k}-1\right)}{p^{k+1}-2 p+1}\right\}+O\left(\frac{\sigma_{-1+\varepsilon}^{*}(n) \delta(x)}{\left.x^{k-1}\right)}\right) .
$$

Lemma 2.8 (Suryanarayana and Sita Rama Chandra Rao (1973; Theorem 4.1)). If $\tau(m, n)$ is the number of divisors of $m$ which are prime to $n$, then for $x \geqq 2$,

$$
\sum_{m \leq x} \tau(m ; n)=\frac{\phi(n) x}{n}(\log x+2 r-1+\alpha(n))+O\left(\sigma_{-\alpha}^{*}(n) x^{\alpha}\right),
$$

where $\gamma$ is Euler's constant, $\alpha(n)$ is given by

$$
\alpha(n)=-\frac{n}{\phi(n)} \sum_{d \mid n} \frac{\mu(d) \log d}{d}=\sum_{p \mid n} \frac{\log p}{p-1},
$$


and $\alpha$ is the number which appears in the Dirichlet divisor problem namely

$$
\sum_{m \leqq x} \tau(m)=x(\log x+2 r-1)+O\left(x^{\alpha}\right) .
$$

It is known that $\frac{1}{4}<\alpha<\frac{1}{3}$ (Hardy and Wright (1960; page 272)). The best result known so far is due to Kolesnik (1969), who proved that the error term in (2.19) is $O\left(x^{12 / 37+\varepsilon}\right)$ for every $\varepsilon>0$. There is a conjecture that $\alpha=\frac{1}{4}+\varepsilon$.

Lemma 2.9. For $x \geqq 3$,

$$
\sum_{m \leqq x} \frac{\mu^{*}(m) \phi(m) \alpha(m)}{m^{k+1}}=-\alpha_{k} \sum_{p} \frac{\log p}{p^{k+1}-2 p+1}+O\left(\frac{\delta(x) \log x}{x^{k-1}}\right) .
$$

Proof. We have by (2.18),

$$
\begin{aligned}
\sum_{m \leq x} \frac{\mu^{*}(m) \phi(m) \alpha(m)}{m^{k+1}} & =\sum_{m \leqq x} \frac{\mu^{*}(m) \phi(m)}{m^{k+1}} \sum_{p / m} \frac{\log p}{p-1} \\
& =\sum_{p d \leq x} \frac{\mu^{*}(p d) \phi(p d) \log p}{p^{k+1} d^{k+1}(p-1)} \\
& =\sum_{\substack{p d \leq x \\
p \mid d}} \frac{\mu^{*}(p d) \phi(p d) \log p}{p^{k+1} d^{k+1}(p-1)}+\sum_{\substack{p d \leq x \\
p \nmid d}} \frac{\mu^{*}(p d) \phi(p d) \log p}{p^{k+1} d^{k+1}(p-1)} \\
& =A+B, \text { say. }
\end{aligned}
$$

We have $\mu^{*}(p d)=\mu^{*}(d)$ and $\phi(p d)=p \phi(d)$ when $p \mid d$. Hence

$$
\begin{aligned}
A & =\sum_{\substack{p d x \\
p \mid d}} \frac{\mu^{*}(d) \phi(d) \log p}{p^{k} d^{k+1}(p-1)} \\
& =\sum_{p d \leq x} \frac{\mu^{*}(d) \phi(d) \log p}{p^{k} d^{k+1}(p-1)}-\sum_{\substack{p d \leq x \\
(p, d)=1}} \frac{\mu^{*}(d) \phi(d) \log p}{p^{k} d^{k+1}(p-1)} \\
& =A_{1}-A_{2}, \quad \text { say. }
\end{aligned}
$$

We have by (2.14),

$$
\begin{aligned}
A_{1} & =\sum_{p \leqq x} \frac{\log p}{p^{k}(p-1)} \sum_{d \leq x / p} \frac{\mu^{*}(d) \phi(d)}{d^{k+1}} \\
& =\sum_{p \leqq x}^{p \leqq x} \frac{\log p}{p^{k}(p-1)}\left\{\alpha_{k}+O\left(\frac{\delta\left(\frac{x}{p}\right)}{\left(\frac{x}{p}\right)^{k-1}}\right)\right\} \\
& =\alpha_{k} \sum_{p} \frac{\log p}{p^{k}(p-1)}-\alpha_{k} \sum_{p>x} \frac{\log p}{p^{k}(p-1)}+O\left(\frac{1}{x^{k-1}} \sum_{p \leq x} \frac{\log p}{p(p-1)} \delta\left(\frac{x}{p}\right)\right) .
\end{aligned}
$$


By (2.1), we have $\alpha_{k}<1$ and $p^{k}(p-1) \geqq p^{k+1} / 2$, so that the second term in (2.23) is

$$
O\left(\sum_{p>x} \frac{\log p}{p^{k+1}}\right)=O\left(\frac{\log x}{x^{k}}\right)=O\left(\frac{\delta(x) \log x}{x^{k-1}}\right)
$$

Also the $O$-term in $(2.23)$ is

$$
O\left(\frac{1}{x^{k}} \sum_{p \leqq x} \frac{\log p}{p} \cdot\left(\frac{x}{p}\right) \delta\left(\frac{x}{p}\right)\right)=O\left(\frac{x \delta(x)}{x^{k}} \sum_{p \leqq x} \frac{\log p}{p}\right)=O\left(\frac{\delta(x) \log x}{x^{k-1}}\right),
$$

since $x \delta(x)$ is monotonic increasing and

$\sum_{p \leqq x} \frac{\log p}{p}=O(\log x)($ Hardy and Wright $(1960 ;$ Theorem 425)).

Hence

$$
A_{1}=\alpha_{k} \sum_{p} \frac{\log p}{p^{k}(p-1)}+O\left(\frac{\delta(x) \log x}{x^{k-1}}\right)
$$

We have by (2.16) and (2.22),

$$
\begin{aligned}
A_{2}= & \sum_{p \leq x} \frac{\log p}{p^{k}(p-1)} \sum_{\substack{d \leq x / p \\
(d . p)=1}} \frac{\mu^{*}(d) \phi(d)}{d^{k+1}} \\
= & \sum_{p \leq x} \frac{\log p}{p^{k}(p-1)}\left\{\alpha_{k} \frac{p\left(p^{k}-1\right)}{p^{k+1}-2 p+1}+O\left(\frac{\delta\left(\frac{x}{p}\right)}{\left(\frac{x}{p}\right)^{k-1}}\right)\right\} \\
= & \alpha_{k} \sum_{p} \frac{\left(p^{k}-1\right) \log p}{p^{k-1}(p-1)\left(p^{k+1}-2 p+1\right)}-\alpha_{k} \sum_{p>x} \frac{\left(p^{k}-1\right) \log p}{p^{k-1}(p-1)\left(p^{k+1}-2 p+1\right)} \\
& +O\left(\frac{1}{x^{k-1}} \sum_{p \leq x} \frac{\log p}{p(p-1)} \delta\left(\frac{x}{p}\right)\right) .
\end{aligned}
$$

By (2.1), we have $\alpha_{k}<1$ and $p-1 \geqq p / 2,\left(p^{k+1}-2 p+1\right)>p^{k+1} / 2,\left(p^{k}-1\right)<p^{k}$, so that the second term is $(2.25)$ is

$$
O\left(\sum_{p>x} \frac{\log p}{p^{k+1}}\right)=O\left(\frac{\log x}{x^{k}}\right)=O\left(\frac{\delta(x) \log x}{x^{k-1}}\right) .
$$

Also, the $O$-term in $(2.25)$ is $O\left(\delta(x) \log x / x^{k-1}\right)$, since it is the same as the $O$-term in (2.23).

Hence

$$
A_{2}=\alpha_{k} \sum_{p} \frac{\left(p^{k}-1\right) \log p}{p^{k-1}(p-1)\left(p^{k+1}-2 p+1\right)}+O\left(\frac{\delta(x) \log x}{x^{k-1}}\right) .
$$

Also, by (2.21) and (2.16), 
(2.27)

$$
\begin{aligned}
B & =\sum_{\substack{p d \leq x \\
(p, d)=1}} \frac{\mu^{*}(p d) \phi(p d) \log p}{p^{k+1} d^{k+1}(p-1)}=-\sum_{\substack{p d \leq x \\
(p, d)=1}} \frac{\mu^{*}(d) \phi(d) \log p}{p^{k+1} d^{k+1}} \\
& =-\sum_{p \leq x} \frac{\log p}{p^{k+1}} \sum_{\substack{d \leq x / p \\
(d, p)=1}} \frac{\mu^{*}(d) \phi(d)}{d^{k+1}}
\end{aligned}
$$

$$
\begin{aligned}
= & -\sum_{p \leq x} \frac{\log p}{p^{k+1}}\left\{\alpha_{k} \frac{p\left(p^{k}-1\right)}{p^{k+1}-2 p+1}+O\left(\frac{\delta\left(\frac{x}{p}\right)}{\left(\frac{x}{p}\right)^{k-1}}\right)\right\} \\
= & -\alpha_{k} \sum_{p} \frac{\left(p^{k}-1\right) \log p}{p^{k}\left(p^{k+1}-2 p+1\right)}+\alpha_{k} \sum_{p>x} \frac{\left(p^{k}-1\right) \log p}{p^{k}\left(p^{k+1}-2 p+1\right)} \\
& +O\left(\frac{1}{x^{k-1}} \sum_{p \leq x} \frac{\log p}{p^{2}} \delta\left(\frac{x}{p}\right)\right) .
\end{aligned}
$$

By (2.1), we have $\alpha_{k}<1$ and $\left(p^{k+1}-2 p+1\right)>p^{k+1} / 2,\left(p^{k}-1\right)<p^{k}$, so that the second term in (2.27) is

$$
O\left(\sum_{p>x} \frac{\log p}{p^{k+1}}\right)=O\left(\frac{\log p}{x^{k}}\right)=O\left(\frac{\delta(x) \log x}{x^{k-1}}\right) .
$$

Also, the $O$-term in $(2.27)$ is $O\left(\delta(x) \log x / x^{k-1}\right)$, since it is the same as the $O$-term in (2.23).

Hence

$$
B=-\alpha_{k} \sum_{p} \frac{\left(p^{k}-1\right) \log p}{p^{k}\left(p^{k+1}-2 p+1\right)}+O\left(\frac{\delta(x) \log x}{x^{k-1}}\right) .
$$

Hence by $(2.21),(2.22),(2.24),(2.26)$ and $(2.28)$, we have

$$
\begin{aligned}
\sum_{m \leq x} \frac{\mu^{*}(m) \phi(m) \alpha(m)}{m^{k+1}}= & \alpha_{k} \sum_{p} \frac{\log p}{p^{k}(p-1)}-\alpha_{k} \sum_{p} \frac{\left(p^{k}-1\right) \log p}{p^{k-1}(p-1)\left(p^{k+1}-2 p+1\right)} \\
& -\alpha_{k} \sum_{p} \frac{\left(p^{k}-1\right) \log p}{p^{k}\left(p^{k+1}-2 p+1\right)}+O\left(\frac{\delta(x) \log x}{x^{k-1}}\right) \\
= & -\alpha_{k} \sum_{p} \frac{\log p}{p^{k+1}-2 p+1}+O\left(\frac{\delta(x) \log x}{x^{k-1}}\right)
\end{aligned}
$$

and the lemma is proved.

LеммA 2.10. For $x \geqq 3$ and for every $\varepsilon>0$,

$$
\sum_{n \leq x} \mu^{*}(n) \tau(m ; n)=O(\chi(m) x \delta(x))
$$

where $\chi(m)=\sum_{d / m} 4^{v(d)}$. 
ProOF. Let

$$
M_{(n)}^{*}(x)=\sum_{\substack{m \leq x \\ n \mid m}} \mu^{*}(m) .
$$

Then we have

$$
\begin{aligned}
\sum_{d \mid n} \mu(d) M_{d}^{*}(x) & =\sum_{d \mid n} \mu(d) \sum_{\substack{m \leq x \\
(m, d)=1}} \mu^{*}(m) \\
& =\sum_{\substack{m \leq x \\
d \mid n \\
(m, d)=1}} \mu(d) \mu^{*}(m)=\sum_{m \leq x} \mu^{*}(m) \sum_{\substack{d \mid n \\
(d, m)=1}} \mu(d) .
\end{aligned}
$$

If $n$ is square-free, then it is easy to show that

$$
\sum_{\substack{d / n \\(d, m)=1}} \mu(d)=1 \text { or } 0,
$$

according as $n \mid m$ or $n \nless m$.

Hence, if $n$ is square-free, then we have

$$
\sum_{d \mid n} \mu(d) M_{d}^{*}(x)=\sum_{\substack{m \leq x \\ n \mid m}} \mu^{*}(m)=M_{(n)}^{*}(x),
$$

and so by (2.3),

$$
\begin{aligned}
M_{(n)}^{*}(x) & =O\left(\sum_{d \mid n} \mu^{2}(d) \sigma_{+1+\varepsilon}^{*}(d) x \delta(x)\right) \\
& =O\left(x \delta(x) \prod_{p \mid n}\left\{1+\sigma_{-1+\varepsilon}^{*}(p)\right\}\right)=O\left(x \delta(x) \prod_{p \mid n} 3\right) \\
& =O\left(3^{\nu(n)} x \delta(x)\right) .
\end{aligned}
$$

Now,

$$
\begin{aligned}
\sum_{n \leq x} \mu^{*}(n) \tau(m ; n) & =\sum_{n \leq x} \mu^{*}(n) \sum_{\substack{d \delta=m \\
(d, n)=1}} 1=\sum_{n \leq x} \mu^{*}(n) \sum_{d \delta=m} \sum_{r(d, n)} \mu(r) \\
& =\sum_{\substack{n \leq x \\
r s \delta=m \\
r \mid n}} \mu^{*}(n) \mu(r)=\sum_{r s \mid m} \mu(r) \sum_{\substack{n \leq x \\
r \mid n}} \mu^{*}(n) \\
& =\sum_{r s \mid m} \mu(r) M_{(r)}^{*}(x) .
\end{aligned}
$$

Hence, for square-free $r$, applying (2.30), we get 


$$
\sum_{n \leqq x} \mu^{*}(n) \tau(m ; n)=O\left(x \delta(x) \sum_{r s \mid m} \mu^{2}(r) 3^{\nu(r)}\right) .
$$

Now the lemma follows, since

$$
\begin{aligned}
\sum_{r s \mid m} \mu^{2}(r) 3^{\nu(r)} & =\sum_{u \mid m} \sum_{r \mid u} \mu^{2}(r) 3^{\nu(r)}=\sum_{u \mid m}\left\{\prod_{\rho \mid u}(1+3)\right\} \\
& =\sum_{u \mid m} 4^{\nu(u)}=\chi(m) .
\end{aligned}
$$

LEMmA 2.11. (Suryanarayana and Sita Rama Chandra Rao (1975; Lemma 2.16)). If the Riemann hypothesis is true, then for $x \geqq 3$ and for every $\varepsilon>0$,

$$
M_{n}^{*}(x) \equiv \sum_{\substack{m \leq x \\(m, n)=1}} \mu^{*}(m)=O\left(\sigma_{-\frac{1}{2}+\varepsilon}^{*}(n) x^{\frac{1}{2}} w(x) \log x\right)
$$

where

$$
\omega(x)=\exp \left\{A \log x(\log \log x)^{-1}\right\},
$$

A being a positive constant.

Lemma 2.12 (Suryanarayana and Sita Rama Chandra Rao (to appear; Lemma 4.3)). If the Riemann hypothesis is true, then for $x \geqq 3$ and for every $\varepsilon>0$,

$$
N_{n}^{*}(x) \equiv \sum_{\substack{m \leq x \\(m, n)=1}} \mu^{*}(m) \phi(m)=O\left(\sigma_{-\frac{1}{2}+\varepsilon}^{*}(n) x^{\frac{1}{2}} \omega(x) \log x\right) .
$$

Lемма 2.13. If the Riemann hypothesis is true, then for $x \geqq 3, s>2$ and for every $\varepsilon>0$,

$$
\sum_{\substack{m>x \\(m, n)=1}} \frac{\mu^{*}(m) \phi(m)}{m^{s}}=O\left(\frac{\sigma_{-\frac{1}{2}+\varepsilon}^{*}(n) \omega(x) \log x}{x^{s-3 / 2}}\right) .
$$

Proof. We get this lemma by following the same argument as in Lemma 2.4 and making use of Lemma 2.12 instead of Lemma 2.3. We have only to replace $\sigma_{-1+\varepsilon}^{*}(n) \delta(x)$ in Lemma 2.4 by

$$
\sigma_{-\frac{1}{2}+\varepsilon}^{*_{1}}(n) x^{-\frac{1}{2}} \omega(x) \log x .
$$

Similarly we get, as in Lemma 2.5 , the following.

LEMMA 2.14. If the Riemann hypothesis is true, then for $x \geqq 3, s>2$ and for every $\varepsilon>0$, 


$$
\sum_{m>x} \frac{\mu^{*}(m) \phi(m) \log m}{m^{s}}=O\left(\frac{\sigma_{-\frac{1}{2}+\varepsilon}^{*}(n) \omega(x) \log ^{2} x}{x^{s-3 / 2}}\right) .
$$

The results corresponding to (2.14), (2.15) and (2.16) in case the Riemann hypothesis is true are given by the following:

$$
\begin{aligned}
& \sum_{m \leq x} \frac{\mu^{*}(m) \phi(m)}{m^{k+1}}=\alpha_{k}+O\left(\frac{\omega(x) \log x}{x^{k-1 / 2}}\right) \\
& \sum_{m \leq x} \frac{\mu^{*}(m) \phi(m) \log m}{m^{k-1}}=-\alpha_{k}\left\{\frac{\zeta^{\prime}(k)}{\zeta(k)}+\sum_{p} \frac{(2 p-1) \log p}{\left(p^{k+1}-2 p+1\right)}\right\} \\
& +O\left(\frac{\omega(x) \log ^{2} x}{x^{k-1 / 2}}\right)
\end{aligned}
$$

$$
\sum_{\substack{m \leq x \\(m, n)=1}} \frac{\mu^{*}(m) \phi(m)}{m^{k+1}}=\alpha_{\iota} \prod_{p \mid n}\left\{\frac{p\left(p^{k}-1\right)}{p^{k+1}-2 p+1}\right\}+O\left(\frac{\sigma_{-\frac{1}{2}+\varepsilon}^{*}(n) \omega(x) \log x}{x^{k-1 / 2}}\right) .
$$

Lемма 2.15. If the Riemann hypothesis is true, then for $x \geqq 3$,

$$
\sum_{m \leqq x} \frac{\mu^{*}(m) \phi(m) \alpha(m)}{m^{k+1}}=-\alpha_{k} \sum_{p} \frac{\log p}{\left(p^{k+1}-2 p+1\right)}+O\left(\frac{\omega(x) \log ^{2} x}{x^{k-1 / 2}}\right) .
$$

Proof. Following the same argument adopted in Lemma 2.9 and making use of (2.38) instead of (2.16), we get this lemma. We have only to replace $\delta(x)$ in Lemma 2.9 by $x^{-\frac{1}{2}} \omega(x) \log x$.

Similarly we get, as in Lemma 2.10, and making use of (2.31) instead of (2.3), the following.

Lемма 2.16. If the Riemann hypothesis is true, then for $x \geqq 3$ and for every $\varepsilon>0$

$$
\sum_{n \leqq x} \mu^{*}(n) \tau(m ; n)=O\left(\chi(m) x^{\frac{1}{2}} \omega(x) \log x\right)
$$

\section{Proof of Theorem 1}

Let $q_{k}^{*}(n)$ denote the characteristic function of the set of unitarily $k$-free integers. It has been shown by Cohen $(1961 ; 3.7$ and 3.1 as $r \rightarrow \infty$ ) that

Hence

$$
q^{*}(n)=\sum_{\substack{d^{k} \delta=n \\(d, \delta)=1}} \mu^{*}(d)
$$

$$
\begin{aligned}
\tau_{(k)}^{*}(n) & =\sum_{r s=n} q^{*}(r)=\sum_{r s=n} \sum_{\substack{d^{k} \delta=r \\
(d, \delta)=1}} \mu^{*}(d)=\sum_{\substack{d^{k} \delta s=n \\
(d, \delta)=1}} \mu^{*}(d) \\
& =\sum_{d^{k} u=n} \mu^{*}(d) \sum_{\substack{\delta s=u \\
(\delta, d)=1}} 1=\sum_{d^{k} u=n} \mu^{*}(d) \tau(u ; d) .
\end{aligned}
$$


Hence

$$
\sum_{n \leq x} \tau_{(k)}^{*}(n)=\sum_{n \leqq x} \sum_{d^{k} u=n} \mu^{*}(d) \tau(u ; d)=\sum_{d^{k} u \leq x} \mu^{*}(d) \tau(u ; d),
$$

the summation on the right being taken over all ordered pairs $(d, u)$ such that $d^{k} u \leqq x$.

Let $z=x^{1 / k}$. Further, let $0<\rho=\rho(x)<1$, where the function $\rho(x)$ will be suitably chosen later.

Now, if $d^{k} u \leqq x$, then both $d>\rho z$ and $u>\rho^{-k}$ can not simultaneously hold, and so from (3.1), we have

$$
\begin{aligned}
\sum_{n \leq x} \tau_{(k)}^{*}(n)= & \sum_{\substack{d^{k} \leq x \\
d \leq \rho z}} \mu^{*}(d) \tau(u ; d)+\sum_{\substack{d^{k} u \leq x \\
u \leq \rho^{-k}}} \mu^{*}(d) \tau(u ; d) \\
& -\sum_{\substack{d \leq \rho_{-} \\
\delta \leq \rho_{-k}}} \mu^{*}(d) \tau(u ; d)=S_{1}+S_{2}-S_{3}, \quad \text { say. }
\end{aligned}
$$

By (2.17), we have

$$
\begin{aligned}
S_{1}= & \sum_{\substack{d^{k} u \leq x \\
d \leq \rho z}} \mu^{*}(d) \tau(u ; d)=\sum_{d \leq \rho z} \mu^{*}(d) \sum_{u \leq x \mid d^{k}} \tau(u ; d) \\
= & \sum_{d \leq \rho z} \mu^{*}(d)\left\{\frac{\phi(d)}{d} \cdot \frac{x}{d^{k}}\left(\log \frac{x}{d^{k}}+2 \gamma-1+\alpha(d)\right)\right. \\
& \left.+O\left(\sigma_{-\alpha}^{*}(d) \cdot \frac{x^{\alpha}}{d^{k \alpha}}\right)\right\} \\
= & x(\log x+2 \gamma-1) \sum_{d \leq \rho z} \frac{\mu^{*}(d) \phi(d)}{d^{k+1}}-k x \sum_{d \leq \rho z} \frac{\mu^{*}(d) \phi(d) \log d}{d^{k+1}} \\
& +x \sum_{d \leq \rho z} \frac{\mu^{*}(d) \phi(d) \alpha(d)}{d^{k+1}}+E_{(k)}^{*}(x),
\end{aligned}
$$

where

$$
E_{(k)}^{*}(x)=O\left(x^{\alpha} \sum_{d \leq \rho z} \frac{\sigma_{-\alpha}^{*}(d)}{d^{k \alpha}}\right) .
$$

If $k=2$ or 3 , then since $\frac{1}{4}<\alpha<\frac{1}{3}$, we have $k \alpha<1$, so that by (2.2),

$$
E_{(k)}^{*}(x)=O\left(x^{\alpha}(\rho z)^{1-k \alpha}\right)=O\left(\rho^{1-k \alpha} z\right) ;
$$

and if $k \geqq 4$, then $k \alpha>1$, so that $E_{(k)}^{*}(x)=O\left(x^{\alpha}\right)$.

Hence we have

$$
E_{(k)}^{*}(x)=O\left(\rho^{1-k \alpha} z\right) \text { or } O\left(x^{\alpha}\right),
$$

according as $k=2,3$ or $k \geqq 4$. 
Naw, by (3.3), (2.14), (2.15) and (2.20), we have

$$
\begin{aligned}
S_{1}= & x(\log x+2 \gamma-1)\left\{\alpha_{k}+O\left(\frac{\delta(\rho z)}{(\rho z)^{k-1}}\right)\right\} \\
& -k x\left\{-\alpha_{k}\left[\frac{\zeta^{\prime}(k)}{\zeta(k)}+\sum_{p} \frac{(2 p-1) \log p}{\left(p^{k+1}-2 p+1\right)}\right]+O\left(\frac{\delta(\rho z) \log (\rho z)}{(\rho z)^{k-1}}\right)\right\} \\
& +x\left\{-\alpha_{k} \sum_{p} \frac{\log p}{\left(p^{k+1}-2 p+1\right)}+O\left(\frac{\delta(\rho z) \log (\rho z)}{(\rho z)^{k-1}}\right)\right\}+E_{(k)}^{*}(x) \\
= & \alpha_{k} x\left(\log x+2 \gamma-1+\frac{\zeta^{\prime}(k)}{\zeta(k)}+\sum_{p} \frac{(2 k p-k-1) \log p}{\left(p^{k+1}-2 p+1\right)}\right) \\
& +O\left(\rho^{1-k} z \delta(\rho z) \log z\right)+E_{(k)}^{*}(x) .
\end{aligned}
$$

We have by (2.29),

$$
\begin{aligned}
S_{2} & =\sum_{\substack{d^{k} \leq \leq x \\
u \leq \rho^{-k}}} \mu^{*}(d) \tau(u ; d)=\sum_{u \leq \rho^{-k}} \sum_{\sqrt[k]{x / u}} \mu^{*}(d) \tau(u ; d) \\
& =O\left(\sum_{u \leq \rho^{-k}} \chi(u)\left(\sqrt[k]{\frac{x}{u}}\right) \delta\left(\sqrt[k]{\frac{x}{u}}\right)\right) .
\end{aligned}
$$

Since $\delta(x)$ is monotonic decreasing and $(\sqrt[k]{x / u}) \geqq \rho z$ we have $\delta(\sqrt[k]{x / u}) \leqq$ $\delta(\rho z)$. Also,

$$
\sum_{n \leq x} \chi(n)=O\left(x \log ^{4} x\right)
$$

Hence

$$
\begin{aligned}
S_{2} & =O\left(z \delta(\rho z) \sum_{u \leq \rho^{-k}} \chi(u) u^{-1 / k}\right)=O\left(z \delta(\rho z)\left(\rho^{-k}\right)^{1-1 / k} \log ^{4}\left(\rho^{-k}\right)\right) \\
& =O\left(\rho^{1-k} z \delta(\rho z) \log ^{4}\left(\frac{1}{\rho}\right)\right) .
\end{aligned}
$$

Also, we have by (2.29),

$$
\begin{aligned}
S_{3} & =\sum_{\substack{d \leq \rho \rho z \\
u \leq \rho-k}} \mu^{*}(d) \tau(u ; d)=\sum_{u \leq \rho-k} \sum_{d \leq \rho \rho z} \mu^{*}(d) \tau(u ; d) \\
& =O\left(\sum_{u \leq \rho-k} \chi(u) \rho z \delta(\rho z)\right)=O\left(\rho z \delta(\rho z) \rho^{-k} \log ^{4}\left(\rho^{-k}\right)\right) \\
& =O\left(\rho^{1-k} z \delta(\rho z) \log ^{4}\left(\frac{1}{\rho}\right)\right) .
\end{aligned}
$$

Hence, by (3.2), (3.5), (3.6) and (3.7), we have 


$$
\begin{aligned}
\sum_{n \leqq x} \tau_{(k)}^{*}(n)= & \alpha_{k} x\left(\log x+2 \gamma-1+\frac{\zeta^{\prime}(k)}{\zeta(k)}+\sum_{p} \frac{(2 k p-k-1) \log p}{\left(p^{k+1}-2 p+1\right)}\right) \\
& +O\left(\rho^{1-k} z \delta(\rho z) \log z\right)+O\left(\rho^{1-k} z \delta(\rho z) \log ^{4}\left(\frac{1}{\rho}\right)\right) \\
& +E_{(k)}^{*}(x) .
\end{aligned}
$$

Now, we choose

$$
\rho=\rho(x)=\left\{\delta\left(x^{1 / 2 k}\right)\right\}^{1 / k},
$$

and write

(3.10) $f(x)=\log ^{\frac{3}{5}}\left(x^{1 / 2 k}\right)\left\{\log \log \left(x^{1 / 2 k}\right)\right\}^{-\frac{1}{5}}=\left(\frac{1}{2 k}\right)^{\frac{3}{5}} U^{\frac{3}{5}}(V-\log 2 k)^{-\frac{1}{3}}$,

where $U=\log x$ and $V=\log \log x$.

$$
\text { For } V \geqq 2 \log 2 k \text {, that is, } U \geqq 4 k^{2}, x \geqq \exp \left(4 k^{2}\right) \text {, }
$$

we have

$$
V^{-\frac{1}{5}} \leqq(V-\log 2 k)^{-\frac{1}{5}} \leqq\left(\frac{V}{2}\right)^{-\frac{1}{5}}
$$

and therefore

$$
\frac{1}{2} k^{-\frac{3}{5}} U^{\frac{3}{5}} V^{-\frac{1}{3}} \leqq f(x) \leqq k^{-\frac{3}{5}} U^{\frac{3}{5}} V^{-\frac{1}{5}} .
$$

We assume without loss of generality that in (2.4)

$$
A<1 \text {. }
$$

By (3.9), (2.4) and (3.10), we have

$$
\rho=\exp \left\{-\frac{A}{k} f(x)\right\} .
$$

By (3.11), we have

$$
k^{-\frac{8}{3}} U^{\frac{3}{5}} V^{-\frac{1}{5}} \leqq \frac{U}{2 k}
$$

Hence by (3.12), (3.13), (3.14) and the above,

$$
\begin{aligned}
\rho & \geqq \exp \left(-A k^{-\frac{k}{3}} U^{\frac{3}{5}} V^{-\frac{1}{3}}\right) \geqq \exp \left(-k^{-\frac{3}{3}} U^{\frac{3}{3}} V^{-\frac{1}{3}}\right) \\
& \geqq \exp \left(-\frac{U}{2 k}\right)=\exp \left(-\frac{\log x}{2 k}\right),
\end{aligned}
$$

So that $\rho \geqq x^{-1 / 2 k}$. 
Hence

$$
\log \left(\frac{1}{\rho}\right) \leqq \log (\sqrt{z})=O(\log x) \quad \text { and } \quad \rho z \geqq x^{1 / 2 k}
$$

Since $\delta(x)$ is monotonic decreasing, $\delta(\rho z) \leqq \delta\left(x^{1 / 2 k}\right)$, so that by (3.12) and (3.14), we have

$$
\rho^{1-k} \delta(\rho z) \leqq \rho \leqq \exp \left\{-\frac{A}{2} k^{-\frac{1}{3}} U^{\frac{3}{3}} V^{-\frac{1}{5}}\right\}
$$

Hence, by (3.15) and (3.16), the first and second $O$-terms of (3.8) are

$$
O\left(x^{1 / k} \exp \left\{-\frac{A}{2} k^{-\frac{8}{5}} U^{\frac{3}{3}} V^{-\frac{1}{3}}\right\} \log ^{4} x\right) .
$$

Hence, if $\Delta_{(k)}^{*}(x)$ denotes the error term in the asymptotic formula (3.8), then we have

$$
\Delta_{(k)}^{*}(x)=O\left(x^{1 / k} \exp \left\{-\frac{A}{2} k^{-\frac{8}{5}} U^{\frac{3}{5}} V^{-\frac{1}{5}}\right\} \log ^{4} x\right)+E_{(k)}^{*}(x)
$$

Case $k=2$ or 3 . In this case, we have $0<1-k \alpha<1$, since $\frac{1}{4}<\alpha<\frac{1}{3}$. By (3.14) and (3.12), we have

$$
\rho^{1-k \alpha}=\exp \left\{-\frac{A(1-k \alpha)}{k} f(x)\right\} \leqq \exp \left\{-\frac{A(1-k \alpha)}{2} k^{-\frac{8}{5}} U^{\frac{3}{5}} V^{-\frac{1}{5}}\right\},
$$

so that by $(3.4)$,

$$
E_{(k)}^{*}(x)=O\left(x^{1 / k} \exp \left\{-\frac{A(1-k \alpha)}{2} k^{-\frac{8}{5}} U^{\frac{3}{5}} V^{-\frac{1}{5}}\right\}\right) .
$$

Again, since $0<1-k \alpha<1$, the first $O$-term in (3.17) is also of the above order of $E_{(k)}^{*}(x)$. Hence

$$
\Delta_{(k)}^{*}(x)=O\left(x^{1 / k} \exp \left\{-B \log ^{\frac{3}{5}} x(\log \log x)^{-\frac{1}{5}}\right\}\right),
$$

where $B$ is a positive constant.

Case $k \geqq 4$. In this case, by (3.4), $E_{(k)}^{*}(x)=O\left(x^{\alpha}\right)$ and the first $O$-term in (3.17) is $O\left(x^{1 / k}\right)=O\left(x^{\frac{1}{4}}\right)=O\left(x^{\alpha}\right)$. Hence $\Delta_{(k)}^{*}(x)=O\left(x^{\alpha}\right)$. Hence Theorem 1 follows.

\section{Proof of Theorem 2}

Following the same procedure adopted in Theorem 1 and making use of (2.36), (2.37), (2.39) and (2.40) instead of (2.14), (2.15), (2.20) and (2.29), we get the following instead of (3.8): 


$$
\begin{aligned}
\sum_{n \leqq x} \tau_{(k)}^{*}(n)= & \alpha_{k} x\left(\log x+2 \gamma-1+\frac{\zeta^{\prime}(k)}{\zeta(x)}+\sum_{p} \frac{(2 k p-k-1) \log p}{\left(p^{k+1}-2 p-1\right)}\right) \\
& +O\left(\rho^{\frac{1}{2}-k} z^{\frac{1}{2}} \omega(\rho z) \log ^{2} z\right) \\
& +O\left(\rho^{\frac{1}{2}-k} z^{\frac{1}{2}} \omega(\rho z) \log z \log ^{4}\left(\frac{1}{\rho}\right)\right)+E_{(k)}^{*}(x) .
\end{aligned}
$$

Now, choosing

$$
\rho=z^{-1 /(1+2 k(1-\alpha))},
$$

we see that $0<\rho<1,1 / \rho<z$, so that $\log (1 / \rho)<\log z$ and

$$
\rho^{\frac{1}{2}-k} z^{\frac{1}{2}}=\rho^{1-k \alpha} z=x^{(2-\alpha) /(1+2 k(1-\alpha))} .
$$

Since $\omega(x)$ is monotonic increasing, we have $\omega(\rho z)<\omega(z)$. Also, by (2.32), we see that $\omega\left(x^{1 / k}\right) \log ^{5} x=O(\omega(x))$. Hence, if $\Delta_{(k)}^{*}(x)$ denotes the error term in the asymptotic formula (4.1), then

$$
\Delta_{(k)}^{*}(x)=O\left(x^{(2-\alpha) /(1+2 k(1-\alpha))} \omega(x)\right)+E_{(k)}^{*}(x) .
$$

Case $k=2$ or 3 . In this case, by (3.4), we have

$$
E_{(k)}^{*}(x)=O\left(\rho^{1-k \alpha} z\right)=O\left(x^{(2-\alpha) /(1+2 k(1-\alpha))}\right) .
$$

Hence by (4.2), Theorem 2 follows in this case.

Case $k \geq 4$. In this case, by (3.4), we have $E_{(k)}^{*}(x)=O\left(x^{\alpha}\right)$. Also, since $k \geqq 4$ and $\frac{1}{4}<\alpha<\frac{1}{3}$, we have

$$
\frac{2-\alpha}{1+2 k(1-\alpha)} \leqq \frac{2-\alpha}{9-8 \alpha}<\alpha .
$$

Since $\omega(x)=O\left(x^{\varepsilon}\right)$ for every $\varepsilon>0$, taking

$$
\varepsilon=\frac{1}{2}\left\{\alpha-\frac{2-\alpha}{9-8 \alpha}\right\},
$$

we see that the first $O$-term in (4.2) is

$$
O\left(x^{\alpha / 2+(2-\alpha) / 2(9-8 \alpha)}\right)=O\left(x^{\alpha}\right)
$$

Hence Theorem 2 follows in this case also. Thus Theorem 2 is completely proved.

\section{References}

E. Cohen (1960), 'Arithmetical functions associated with the unitary divisors of an integer', Math. Z. 74, 66-80.

E. Cohen (1961), 'Some sets of integers related to the $k$-free integers', Acta Sci. Math. (Szeged) 22, 223-233. 
G. H. Hardy and E. M. Wright (1960), An Introduction to the Theory of Numbers (Clarendon Press, Oxford, 4th ed. 1960).

G. A. Kolesnik (1969), 'An improvement of the remainder term in the divisors problem', Mat. Zametki 6, 545-554= Math. Notes 6, 784-791.

D. Suryanarayana and V. Siva Rama Prasad (1973), 'The number of $k$-free and $k$-ary divisors of $m$ which are prime to n., J. Reine Angew Math., 264, 56-75.

D. Suryanarayana and R. Sita Rama Chandra Rao (1975), 'Distribution of unitarily $k$-free integers', J. Austral. Math. Soc. 20, 129-141.

D. Suryanarayana and R. Sita Rama Chandra Rao (to appear), 'The number of bi-unitary divisors of an integer-II', J. Indian Math. Soc.

\section{Department of Mathematics}

Andhra University

Waltair, India. 